\title{
Nurses' Perceived Factors of Reluctance to Implement the Bowel Regimen Protocol in Intensive Care Units
}

\author{
Ghada Shalaby Khalaf Mahran Manal Moammed Abd Elnaeem* \\ Lecturer of Critical Care and Emergency Nursing, Faculty of Nursing, Assiut University, Assiut, Egypt
}

\begin{abstract}
Aims: To identify nurses' perceived factors of reluctance to implement the bowel protocol in the ICUs. Background: However, Bowel regimen protocol of patients in the intensive care unit is safe, feasible, and beneficial, its' implementation as part of routine nursing clinical care can be challenging. Study design: Descriptive cross-sectional design. Methods: A cross-sectional survey of 64 nurses who were working in intensive care units and meet the inclusion criteria was conducted between April 2017 and February 2018. The survey instrument was a semi-structure interview questionnaire developed by the researchers based on the related literature. Results: The mean age of studied nurses was $29.12 \pm 6.41$. The most frequently nurses' perceived factors of reluctance to deliver bowel regimen were the busy shift $(93.8 \%)$, limited staff \& lack of supervision $(92.2 \%)$, the low priority of bowel care $(90.6 \%)$ when considered against other demands of the highly technical intensive care units, unclear order from the doctors $(89.06 \%)$., forgetfulness and lack of education and awareness $(92.2 \%)$. Conclusion: This study provides an understanding of the perceived factors of reluctance to deliver bowel regimen for critically ill patients. Implications for Nursing Management: Nurse Managers could deal with factors of reluctance to deliver bowel regimen protocol in the ICUs and develop new strategies to overcome these factors
\end{abstract}

Keywords: critical care; intensive care; bowel care

DOI: $10.7176 / \mathrm{JHMN} / 73-10$

Publication date: April $30^{\text {th }} 2020$

\section{Introduction}

The regularity and frequency of bowel actions is varied widely in adults. So it is necessary to ascertain the individual's normal habit continuously (Knowles et al., 2014). Disturbance in normal bowel habits always occurs in patients with life threatening problems due to interventions such as enteral feeding intolerance, diarrhoea and constipation (Asai 2007, Ferrie \& East 2007\& Oczkowski et al., 2017). This disturbance can lead more adverse events such as a prolonged period of mechanical ventilator with high frequency of weaning failure, delayed discharge from intensive care units (ICU ) and bowel obstruction(Patrick-Heselton, 2011;Knowles et al., 2014).

There is a variation in the ICU incidence of constipation and diarrhoea: the previous reports for constipation ranging from 16 to $50 \%$ and in one instance even as high as $83 \%$; and with reports for diarrhoea ranging from 15 to $36 \%$ (Knowles et al., 2014). The definition of constipation as ( the inability of patients to pass stool within 72 hours of ICU admission) stated by Working Group on Abdominal Problems of the European Society of Intensive Care Medicine (ESICM) and considered this 72 hour as a cutoff to define "paralysis of the lower gastrointestinal tract" (Blaser et al., 2012);and this definition still used in critical care practice (Vincent J-L Preiser, 2015)

\subsection{Background}

There are various factors for bowel alteration in acutely ill patients. Dehydration is one of these important factors. Moreover, sedatives or analgesics, including opioids were reported as common factors of bowel alteration in various publications (Smonig et al., 2016). Furthermore, immobility; mechanical ventilation; or the underlying illness or disease process can lead to bowel dysfunction (Oczkowski et al., 2017). Both constipation and diarrhoea can lead to undesirable side effects, including disturbance in fluid and electrolyte balance, skin breakdown, wound infection, feeding intolerance, delirium, increased duration of mechanical ventilation, hospital stay and mortality day (Ferrie \& Daley 2011; Rees \& Sharpe 2009), (Gacouin et al. 2010), (Smonig et al., 2016), ( Masri, Abubaker \&Ahmed 2012).

Checking of bowel function regularly and management of bowel disturbance are associated with improved patient outcomes. The responsibilities of this checking lie with each nurse caring for the patient. (Ritchie et al., 2008; Yates, 2011) (McPeake et al., 2011). A bowel regimen protocol is one of the methods that offer a guide to clinicians in evidence based assessment and treatment for bowel function in ICU (Gagliardi \& Brouwers 2012). There are many benefits of using bowel management protocol as patient comfort, saving the nursing time needed to manage every episode of faecal incontinence as well as cost implications for reducing hospital-related infections (Hurnauth, 2011; García et al., 2013), (Peart J Richardson, 2015).

It was reported (Knowles et al., 2010) that often time bowel care is neglected in ICU as the hemodynamic stability is the primary focus of the patients. Furthermore, there is a little nursing research evidence to support efficient nursing bowel care (Rogers, 2008). Therefore, this research aimed to identify nurses' perceived factors that may interfere with the delivery of the bowel regimen protocol in the ICU. 


\section{Methods:}

2.1. Aims: This study aimed to identify nurses' perceived factors of reluctance to implement the bowel protocol in the ICUs.

2.2. Research questions: What are nurses' perceived factors of reluctance to implement the bowel protocol in the ICUs?

2.3. Research design: Descriptive cross-sectional research study was conducted between April 2017 and February 2018.

2.4. Study site: The study site included ICU for trauma patients, general ICU, obstetric ICU and ICU for cardiac patients) at University Hospitals in Assiut, Egypt.

2.5. The study sample: The participants of this study were 64 nurses working in intensive care units and meet the inclusion criteria. Nursing staff were included in the study because nurses spend the most time in direct contact with hospitalized patients (Buckley and Mateucci, 2012; Rice and Stead, 2008). The number of nurses screened in the selected ICUs was 145 nurses. 45 nurses didn't agree to participate in the study, and 36 nurses didn't complete the questionnaire. The final number of the participant nurses was 64. Inclusion criteria: Nurses who provided direct care to critically ill patients. Exclusion criteria: Student nurses and Nurses management facilitators who are not responsible for direct care for critically ill patients.

2.6. Data collection tool: one tool was used in this study to collect the data (a semi-structure interview questionnaire).

2.7. Data collection phases: The study comprised three distinct phases, including (i) Implementation of search of different web sites for the development of a questionnaire to identify factors that limit the implementation of bowel care; (ii) Implementation of pilot testing of the questionnaire; and (iii) Administration of the questionnaire.

2.8. Questionnaire development: The survey questionnaire was developed by the researchers for use in this study based on a review of the related literature (Coggrave et al., 2014;Peart J Richardson, 2015;García et al., 2013;Varghese, 2013). The questionnaire was a self-reporting data collection tool where the participants provide their views about factors to implementing bowel care protocol. It was simple, not time-consuming to enable the researcher to obtain larger samples and assure that patients are not affected. This questionnaire included close ended questions (dichotomous yes/no type question)

2.9. Piloting the questionnaire: Once the approval of the ethical committee was received, the questionnaire was pilot tested. Pilot testing helps the researcher to identify confusing and irrelevant questions that may exist in the questionnaire. A group of 6 nurses was chosen for the pilot study including three registered nurses and three head nurses'. The reason for selecting these nurses was to test the tool with a group which reflected the expected profile of the target population. These nurses were asked to provide feedback and comments about the questionnaire. According to the feedback obtained, there were few changes made to the final questionnaire. Some questions required rewording to reduce ambiguities.

2.10. Validity and Reliability: A panel group of six experts in critical care nursing and medicine received the questionnaire from the researcher via email after taking their informed consent to examine the content of the questionnaire. Those expertise are different from the six nurses on piloting, they were included according to their qualification. The experts received a letter contained the objective of the study and the duration of the analysis of the questionnaire ( 3 weeks) that they had to send their own evaluations after this period. Their modifications were utilized to improve the quality of the questionnaire. The content validity index was 0.88 . The reliability was measured by the correlation coefficient (Cronbach's alpha was 0.90 )

2.11. The final questionnaire: The final form of the questionnaire included a total of twenty-five questions that included two parts. The first part composed of (four questions) related to socio-demographic data (Age, type of ICU, and level of experience and educational level). The second part included twenty one closed ended questions about the factors. These factors were classified as nurses related factors (6 questions), institutional factors (7 questions), culture related factors (4 questions), process related factors (4 questions) (Varghese, 2013).

This final form of the questionnaire was printed and copied (64 copies) to be distributed to the participants. The researcher interviewed with the nurses who accepted to participate in the study and distributed the hard copies and asked them to fill it out and present their views related to the factors. The researchers waited near the 
participants in each ICU to explain any potential questions. The completed copies of the questionnaire were recollected immediately after the nurses finished their comments.

\section{Findings analysis}

SPSS for Windows version 16.00 (SPSS Inc., Chicago, IL, USA) was used for statistical analysis. Descriptive statistics are presented by numbers (n), percentages (\%) and as mean (standard deviation).

\section{Ethical and research approvals:}

The research proposal was submitted for the approval of the study to the ethical committee of faculty of nursing (ethical approval was from the faculty of nursing review board, Number, 600). Each ICU coordinator was informed about this study and gave their verbal support and written consent for the study. Participants were not impelled to participate in the study and had the right to withdraw from the study by not completing the survey.

\section{Results:}

By looking more closely to Table 1, it can be seen that $31.5 \%$ of nurses were working in trauma care unit and coronary care unit. The mean and SD of nurses age was 29.12 \pm 6.41 . Moreover, most nurses had bachelor \& diploma degree with a percentage of $46.87 \%$ \& $39.06 \%$ respectively. Furthermore, $46.87 \%$ of nurses had work experience ranged from (one to two years).

Table (1): Distribution of Socio-demographic characteristics of studied nurses $(n=64)$

\begin{tabular}{|l|l|l|}
\hline Items & Frequency & Percent (\%) \\
\hline Age(mean and SD) & \multicolumn{2}{l|}{$29.12 \pm 6.41$} \\
\hline Department & 20 & 31.25 \\
\hline Trauma intensive care unit. & 17 & 26.5625 \\
\hline General intensive care unit. & 20 & 31.25 \\
\hline Coronary ICU & 7 & 10.9375 \\
\hline Obstetric ICU & \multicolumn{2}{|l|}{} \\
\hline Education & 25 & 39.062 \\
\hline Diploma & 30 & 46.87 \\
\hline bachelor & 9 & 14.06 \\
\hline Master & 0 & 0 \\
\hline PHD & \multicolumn{2}{|l|}{} \\
\hline Level of experience & 30 & 46.875 \\
\hline $1<2$ year & 14 & 21.875 \\
\hline $2<5$ year & 6 & 9.375 \\
\hline $5<10$ year & 14 & 21.875 \\
\hline$>10$ year & \multicolumn{2}{|l|}{} \\
\hline
\end{tabular}

It is evident from Table 2 that there were multiple factors related to nurses' attitude which imped the implementation of bowel regimen as forgetfulness $(82.8 \%)$ as some nurses commented that: "There is no bowel function chart in patient sheet".

Moreover, it's obvious that deficiency of awareness $(81.2 \%)$ about the bowel care was a big barrier of implement bowel protocol. It was reported by some nurses as the main focus in their care of intensive care patients is the maintenance of hemodynamic stability and not common for them to care about issue like bowel care. In addition to, the lack of responsibility was reported $(78.1 \%)$ as a factor to prevent bowel care, some nurses said: "I'm not the only person who responsible for this type of care"

"It should be a combined effort that nurse, doctor, dietician and team leader should be involved" Lack of accountability for neglecting bowel care was reported by some nurses $(62.5 \%)$ due to inadequate supervision of the staff. The nurses lack of concern was reported by $(54.7 \%)$ as a factor that prevent bowel care, some nurses responded: "it is not of our daily basis in nursing care"

Some nurses (42.2\%) reported that unwillingness of the staff to administer the aperients is a real factor that prevents bowel protocol application. Some nurses comments: "it is unpleasant and disgusting to clean up the faeces" "We don't want any bad odor during the shift" "They are always tired due to the long time of the work shift and they don't have the ability to turn the patients during aperients administration for safety" 
Table (2): frequencies and distributions of nurses perceived factors of reluctance to implement the bowel protocol

\begin{tabular}{|c|c|c|}
\hline Items & Frequency & Percent \\
\hline \multicolumn{3}{|l|}{ Nurses attitude related factors } \\
\hline 1. Forgetfulness to bowel care & 53 & 82.8 \\
\hline 2. Lack of concern of bowel care & 35 & 54.7 \\
\hline 3. Un-willingness of the staff to administer aperients & 27 & 42.2 \\
\hline 4. Lack of responsibility of bowel care & 50 & 78.1 \\
\hline 5. Lack of awareness of bowel care protocol & 52 & 81.2 \\
\hline 6. Lack of accountability of neglecting bowel care & 40 & 62.5 \\
\hline
\end{tabular}

Table 3 shows the institutional factors of reluctance of bowel care: Busy shift was the most important (93.8\%) factor that interferes with implementation of bowel care due to the assignment of more than one patient for each nurse, followed by lack of education, supervision and regular checking of bowel care with a percentage (92.2 \%). some nurses comments: "If there is a checking of bowel, the checking occur only at the beginning of each shift"

Moreover, inadequate staff training about bowel care was reported by nurses $(81.2 \%)$ as a big barrier of bowel care. This factor is due to the work over load \& busy shift.

Table (3): frequencies and distributions of Institutional related factors of reluctance to implement the bowel protocol

\begin{tabular}{|c|c|c|}
\hline Items & Frequency & percent \\
\hline \multicolumn{3}{|l|}{ Institutional related factors } \\
\hline 1. Lack of education about bowel care & 52 & 81.2 \\
\hline 2. Lack of supervision or regular checking of bowel care & 59 & 92.2 \\
\hline 3. $\quad$ Limited staff & 59 & 92.2 \\
\hline 4. time constrain & 35 & 54.7 \\
\hline 5. Busy shift & 60 & 93.8 \\
\hline 6. Lack of bowel care guideline poster & 44 & 68.8 \\
\hline 7. Unclear link with existing enteral feeding protocols & 35 & 54.7 \\
\hline
\end{tabular}

Table 4 shows the culture related factors that adherence the bowel care. The perception of nurses as bowel care not priority was the most culture related factor with a percentage of $(90.6 \%)$ followed by lack of bowel care culture with a percentage (75\%). Moreover, nurses' deficiency of awareness about the benefits of bowel care was reported as a big barrier (71.9\%). It was reported by some nurses as the main focus in their care of intensive care patients is the maintenance of hemodynamic stability and not common for them to care about issue like bowel care. Moreover, insufficient supports from the staff were reported by the nurses (57.8\%) as a barrier to implement bowel regimen protocol.

It can be seen that the most process-related factors reported by the nurses to implement the bowel regimen protocol was unclear order or documented role from the doctors to the nurses to implement bowel care, with a percentage (89.06\%).

Table (4): frequencies and distributions of Culture $\&$ process related factors of reluctance to implement the bowel protocol

\begin{tabular}{|l|l|l|}
\hline Items & Frequency & percent \\
\hline Culture related factors & 48 & 75 \\
\hline Lack of bowel care culture & 58 & 90.6 \\
\hline Bowel care not priority & 37 & 57.8 \\
\hline Lack of support & 46 & 71.9 \\
\hline Lack of staff knowledge about benefits of bowel care & \multicolumn{2}{|l|}{} \\
\hline Process related factors & 47 & 73.4 \\
\hline Lack of planning and coordination & 57 & 89.1 \\
\hline Unclear order or role & 52 & 81.2 \\
\hline Missing daily screening & 42 & 65.6 \\
\hline Poor documentation & & \\
\hline
\end{tabular}

\section{Discussion:}

Intensive care setting patients are generally seriously ill, hemodynamically unstable, and have life threating problems or multiple organ dysfunctions. They are connected by different lifesaving high technology machines as mechanically ventilator, pacemakers, medications and other high-risk procedures (Li et al., 2012). When faced with this type of patients, bowel management seems to unintentionally, assume low priority(McPeake et al., 2011). Monitoring and documenting bowel activity is usually within the domain of nurses. In these recent days, bowel assessment has been added to daily checklists for medical officers (Vincent \& Hatton 2009). 
This study is the first one known in Egypt to identify nurses' perceived factors that may interfere with the delivery of bowel regimen protocol in the ICU. The study findings indicate that a majority of nurses reported that the most frequently perceived factors by nurses to deliver bowel regimen were the busy shift, limited staff \& lack of supervision, the low priority of bowel care when considered against other demands of the highly technical intensive care units, unclear order from the doctors, forgetfulness and lack of education and awareness. These findings were highlighted in another study was conducted by (Varghese, 2013) at tertiary teaching hospital in Adelaide in Australia.

The lack of communication between nurses and doctors in the ICU lead to a deficiency in the documentation. This factor occurs due to the higher powerful position of doctors in the ICUs than nurses. A lack of priority and awareness from staff regarding bowel care was also identified in this study. This might be related to the fact that the majority of them were not receiving any previous training about bowel care. This lack of knowledge may also be due to the absence of pre-employment orientation programs. Another cause for lack of knowledge in nurses is the exhaustion due to increased workload which may hinder their ability to read and update their knowledge. Nurses have reported dissatisfaction with the management of bowel function in intensive care patients within their units. These findings were reflected in study of (Knowles et al., 2010). This may be assumed that their priority of care directed to maintaining the hemodynamic stability of the patients rather than bowel care. These findings were in line with (Ritchie et al., 2008) e.g. found that in the ICU bowel care often seems to assume low priority. Inefficient time was a significant barrier that may be explained by a large number of patients and consequently more than one patients assigned to every nurse. These findings were in line with (Peart J Richardson, 2015).

\section{Strength and limitations:}

The survey of this study was conducted in multi-intensive care units within one institution. This design resulted in some strength as better understanding of nurses care concerns around bowel regimen in this location. It is also, resulted in some imitations as limiting generalizability and the low response rate by nursing provider's limits the generalizability of the nursing results.

\section{Implications and recommendations for practice:}

Very few studies have identified the nurses' perceived factors to implement a bowel care protocol of ICU patients. Therefore, further studies are needed to identify additional factors and develop new strategies to overcome these factors.

Further research could explore the doctors' and pharmacists' role in enhancing the delivery of the bowel care protocol when providing holistic nursing care.

\section{Recommendations:}

Further research should be conducted to investigate these factors with an observational method to observe actual practice relating to bowel care and add clarity to the factors affecting the delivery of bowel care. Encourage nurses to attend in-services education and regular up-date with evidence based practice for bowel care. Moreover, further education for all health team regarding the importance of bowel care is recommended. Also, the supervisors of each ICU should assure accountability for nurses' actions. A poster about bowel regimen protocol should be present in each ICU.

\section{Conclusion:}

Regular assessment and management of faecal incontinence is a crucial role of critical care nurses. This Study has identified numerous perceived factors of reluctance to implement bowel care. Systematic efforts to develop efficient strategies to overcome these factors are essential for improvements of critically ill patients' outcomes.

\section{Funding/Support:}

The authors report no conflict of interest during the performance of this study. This research received no specific grants from any funding agencies in the public, commercial, or not-for-profit sector. The authors have no competing interests that may affect the outcome of this study.

\section{Conflict of interest:}

The authors report no conflict of interest during the performance of this study.

\section{Acknowledgement:}

The authors wish to thank the Assiut University Hospital ICUs, Egypt, for their support with participant recruitment and data collection. Also like to thank the study participants for their involvement in the study. 


\section{Authorship:}

Ghada Shalaby Khalaf Mahran : Suggested the idea and have made substantial contributions to conception and design, or acquisition of data, or analysis and interpretation of data

Manal Moammed Abd Elnaeem: Shared in database and analysis \& involved in drafting the manuscript or revising it critically for important intellectual content.

\section{References}

Blaser AR, Malbrain ML, Starkopf J, Fruhwald S, Jakob SM, De Waele J, Braun J-P, Poeze M Spies C. (2012). Gastrointestinal function in intensive care patients: terminology, definitions and management. Recommendations of the ESICM Working Group on Abdominal Problems. Intensive care medicine; 38: $384-$ 394.

Coggrave M, Norton C Cody JD. ( 2014). Management of faecal incontinence and constipation in adults with central neurological diseases.

García CB, Binks R, De Luca E, Dierkes C, Franci A, Gallart E, Niederalt G, Wyncoll D, Vaes P Soderquist B. (2013). Expert recommendations for managing acute faecal incontinence with diarrhoea in the intensive care unit. Journal of the Intensive Care Society; 14: 1-9.

Hurnauth C. (2011). Management of faecal incontinence in acutely ill patients. Nursing Standard (through 2013); 25: 48.

Knowles S, McInnes E, Elliott D, Hardy J Middleton S. (2014). Evaluation of the implementation of a bowel management protocol in intensive care: effect on clinician practices and patient outcomes. Journal of clinical nursing; 23: 716-730.

Knowles S, Rolls K, Elliott D, Hardy J Middleton S. (2010). Patient care guidelines: a telephone survey of intensive care practices in New south Wales. Australian Critical Care; 23: 21-29.

Li B, Wang J-R Ma Y-L. (2012). Bowel sounds and monitoring gastrointestinal motility in critically ill patients. Clinical Nurse Specialist; 26: 29-34.

McPeake J, Gilmour H MacIntosh G. (2011). The implementation of a bowel management protocol in an adult intensive care unit. Nursing in critical care; 16: 235-242.

Oczkowski SJ, Duan EH, Groen A, Warren D Cook DJ. (2017). The Use of Bowel Protocols in Critically Ill Adult Patients: A Systematic Review and Meta-Analysis. Critical care medicine; 45: e718-e726.

Patrick-Heselton J. (2011). Faecal incontinence in critical illness. Nursing times; 107: 23-4, 26.

Peart J Richardson A. (2015). Developing a critical care bowel management assessment tool to manage faecal incontinence. Nursing in critical care; 20: 34-40.

Ritchie G, Burgess L, Mostafa S Wenstone R. (2008). Preventing constipation in critically ill patients. Nursing times; 104: 42-44.

Rogers J. (2008). The IMPACT paediatric bowel care pathway. Nurs Times; 104: 46-47.

Smonig R, Wallenhorst T, Bouju P, Letheulle J, Le Tulzo Y, Tadié JM Gacouin A. (2016). Constipation is independently associated with delirium in critically ill ventilated patients. Intensive care medicine; 42: 126127.

Varghese RJ. (2013). Awareness and attitudes of intensive care nurses regarding the bowel protocol for the critically ill patients.

Vincent J-L Preiser J-C. (2015). Getting critical about constipation. Pract Gastroenterol; 15.

Yates A. (2011). Faecal incontinence: a joint approach to guideline development. Nursing times; 107: 12-14.

Buckley LL, Matteucci R. (2012). Smoking cessation: Nursing interventions in hospitalized patients. CINAHL Plus with full Text - Evidence based care sheets: Glendale. CINAHL information system.

Rice VH, Stead LF. (2008). Nursing interventions for smoking cessation. Cochrane Database of Systematic Reviews; 1. DOI: 10.1002/14651858.CD001188.pub3.

Asai T. (2007) .Constipation: does it increase morbidity and mortality in critically ill patients? Critical Care Medicine 35, 2861-2862.

Ferrie S \& East V. (2007). Managing diarrhoea in intensive care. Australian Critical Care 20, 7-13.

Gagliardi A \& Brouwers M (2012). Integrating guideline development and implementation: analysis of guideline development manual instructions for generating implementation advice. Implementation Science 7, 67.

Gacouin A, Camus C, Gros A, Isslame S, Marque S, Lavoue' S, Chimot L, Donnio P-Y \& Le Tulzo Y. (2010). Constipation in long-term ventilated patients: associated factors and impact on intensive care unit outcomes. Critical Care Medicine 38, 1933-1938.

Masri, Y. Abubaker, J \&Ahmed, R. (2012). Prophylactic use of laxative for contipation in critically ill patients. Annals of Thoracic Medicine, vol.5, no.5,pp.228-231.

Ferrie, S \& Daley, M. (2011). Lactobacillus GG as treatment for diarrhoea during enteral feeding in critical illness: Randomized controlled trial, journal of parenteral and enteral nutrition,vol.35, no.1,pp.43-49.

Rees,J \& Shape, A. (2009). The use of bowel management system in the high-dependency settings.British journal 
of nursing, vol.18, no 7, pp.s19-s24.

Vincent WRr \& Hatton KW. (2009). Critically ill patients need "FAST HUGS BID" (an updated mnemonic). Critical Care Medicine 37, 2326-2327; author reply 2327. 